\title{
Driving Licence Registration and Verification System using Smart Contract
}

\author{
Rishikesh Kale ${ }^{1}$, Abhijeet R. Raipurkar ${ }^{2 *}$ and Manoj B. Chandak ${ }^{3}$ \\ 1,2, ${ }^{3}$ Department of Computer Science and Engineering, Shri Ramdeobaba \\ College of Engineering and Management, Nagpur, India
}

\section{ABSTRACT}

Every kind of business depends on the evolving technologies that tend to take advantages of new opportunities. With every new technology, different set of challenges appear. In recent years, numerous blockchain-based applications have been proposed to solve problems in different areas. In this paper, an adaptive strategy is proposed to eliminate driving license frauds using blockchain technology. With second generation of blockchain technologies, smart contracts have helped to develop trust and automated transactions between large numbers of users. Smart Contracts are programs that run on the blockchain to take action on the records. This paper proposes a driving license registration and verification smart contract using Ethereum blockchain. The smart contract mechanism allows for registration of the officers/agents by a higher authority. It can dynamically restrict unregistered officer/fraud agents from generating fake driving license. Also, the smart contract facilitates verification of a registered driving license which includes checking its validity and authorization. It provides special rights to the traffic regulator officer to add remarks to the driving license if the driving license holder is found violating the traffic rules. Since the blockchain is an immutable ledger, this remark cannot be tampered and the apprehended person would be liable to pay the fines. This would result in reduction of traffic accidents and violation of traffic rules. This smart contract opens up doors for more innovations in this field and improves traffic regulation and management.

\section{KEY WORDS: BLOCKCHAIN; ETHEREUM BLOCKCHAIN; SMART CONTRACT NEIGHBOUR.}

\section{INTRODUCTION}

The traditional system of issuing driving license is centralized and the data is maintained in centralized servers. [Michael Crosby, Pradan Pattanayak, Sanjeev Verma, Vignesh Kalyanaraman, 2016] According to current system, as per the request, organizations are authorized to issue and validate the identities. There are chances of data breaching as under this system, third

\section{ARTICLE INFORMATION}

*Corresponding Author: raipurkarar@rknec.edu

Received 18th Oct 2020 Accepted after revision 30th Dec 2020

Print ISSN: 0974-6455 Online ISSN: 2321-4007 CODEN: BBRCBA

Thomson Reuters ISI Web of Science Clarivate Analytics USA and Crossref Indexed Journal

\section{Clarivate
Analytics}

NAAS Journal Score 2020 (4.31)

A Society of Science and Nature Publication,

Bhopal India 2020. All rights reserved.

Online Contents Available at: http//www.bbrc.in/

Doi: http://dx.doi.org/10.21786/bbrc/13.14/2 parties are responsible for keeping the security of the data. The importance of digital identity is very much important. Due to the lack of proper security issues, current scenario does not guarantee the security of the data. So, many users face identity issues that cause a massive problem in their social life as well. To ensure a proper management of system, all the problems including data security need to be focused and solved in fast track manner.

Blockchain is a secure peer-to-peer, or person-to-person, transaction system for transacting money, or other items of value. [Rajarshi Mitra] The blockchain is considered as immutable ledger, which means that once a data is entered into the blockchain it cannot be tampered or erased. Every node in the distributed and decentralized network holds the copy of the blockchain and can view the data entered into the blockchain, which increase the transparency. The decentralized nature of blockchain prohibits the data from being owned by one centralized

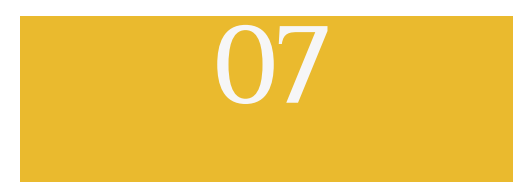


entity. Blockchain run in a "trustless", open environment. So, this allows for transactions to be completed without a 3rd party to provide trust. On the blockchain, if a value needs to be changed, a new entry is added to the ledger, as a transaction, and the value is updated. The new record does not remove the previous records.

1.1 Smart Contract: A smart contract [Fries, Martin; P. Paal, Boris, 24 May, 2020][ Ameer Rosic] is a computer program or a transaction protocol respectively, which is intended to automatically execute, control or document respectively legally relevant events and actions according to the terms of a contract, of an agreement or of a negotiation. The objectives of smart contracts are the reduction of need in trusted intermediates, arbitrations and enforcement costs, fraud losses, as well as the reduction of malicious and accidental exceptions.

1.2 Decentralization: Blockchain is a decentralized system [Raval, Siraj, 2016] because single person cannot control it. Every node in the system has their copy of the blockchain. Nodes are also architecturally decentralized because their infrastructure has no central point of failure, as each node keeps a copy of the blockchain. The data in blockchain is stored across its peer-to-peer network, thus it eliminates a number of risks which come when the data is held centrally. The crackers always target the central points of failure to exploit, however the peer-to-peer blockchain network does not have the centralized points of vulnerability thus provide security from data breach.

2. Literature Survey: Identity is an essential factor in a life of a human being. In our society, daily activities are associated with user's identity. With evolution in technology the traditional system paper-based system is being replaced with digital identity. [Roger Aitken] The idea of a digital identity has several potential benefits, and strengthening it with Blockchain makes it a more fungible and realistic possibility. Blockchain technology provides a decentralized environment and has the ability to transform and replace traditional systems with a highly trusted mechanism which is capable of managing identities. This decentralized environment facilitates the user with greater control over users own identity.

2.1 WEF Known Traveller Digital ID: In collaboration with the WEF, Accenture introduced Known Traveller Digital Identity [The Known Traveller website, January 2018] or KTDI a World Economic Forum initiative which brings together a global consortium of individuals, governments, authorities and the travel industry to enhance security in world travel. It allows the travelers to share documentation and information from one verified identity with partners including government authorities, airlines and hotels. The KTDI allows travelers to play an active role in travel security efforts by sharing their information proactively. Travelers always retain control over what, where and with whom they share their information. Each data element shared by the traveler is verified, accurate and consistent every time. This is made possible by the technologies of distributed ledger, cryptography and biometrics.

2.2 Sovrin: Sovrin [Drummond Reed, Jason Law \& Daniel Hardman, 29 September, 2016] is one of the popular projects offering a self-sovereign identity for the users. In reality, it's a non-profit organization that solely works with decentralized digital identity solutions. With this, user can get personal control, trust, and easy to use digital identities. Basically, it's a metasystem that allows organizations, people the freedom to use any application without worrying about their identities. Moreover, Sovrin also possesses verifiable credentials options.

2.3 Civic: Civic [Vinny Lingham, Jonathan Smith, 2017] is a personal identity verification process that uses blockchain technology to offer digital identities. It proposes an ecosystem, wherein an individual can create his/her own virtual identity along with storing additional information as well. It facilitates on-demand, secure and low-cost access to identity verification services. It requires verification of all credentials of an individual before storing it on the digital identity solutions, however the background and personal information checks will no longer need to be undertaken from ground up every time.

2.4 uPort: ConsenSys developed a self-sovereign identity system, known as uPort, [Dr. Christian Lundkvist, Rouven Heck, Joel Torstensson, Zac Mitton, Michael Sena, 2016] based on Ethereum blockchain. uPort aims to return ownership of digital identity to the individual. It uses blockchain to create an identifier (an Ethereum address) and a public-private key pair for signing transactions. The registry of identities on Ethereum allows for information about an identity - whether that be a degree earned or permission granted to that identity - to be sent to and held by the holder of that identity. The smart contract behind uPort application allows user to create and store personal information and generate their identity, the associated key of the user is held by its mobile application. It assures security and recovery of data in case of loss of device with which the key was linked.

\section{System Design And Development On Ethereum} Blockchain: Ethereum is a global open source fully distributed "peer-to-peer" computer network. Ethereum runs on a distributed network. Each computer on the network is a peer and has a copy of the ledger with all the data. Distributed networks always ensure the blockchain is up and running and thus a single point of failure in network will not affect the whole system. Ethereum has made it possible to exchange any type of asset or information on a blockchain. Ethereum is a second generation blockchain where users can write applications, called Decentralized Applications, or DApps that transact using digital currency, called Ether (ETH). A decentralized application is a computer application that runs on a distributed computing system. 
Figure 1: Ethereum Virtual Machine

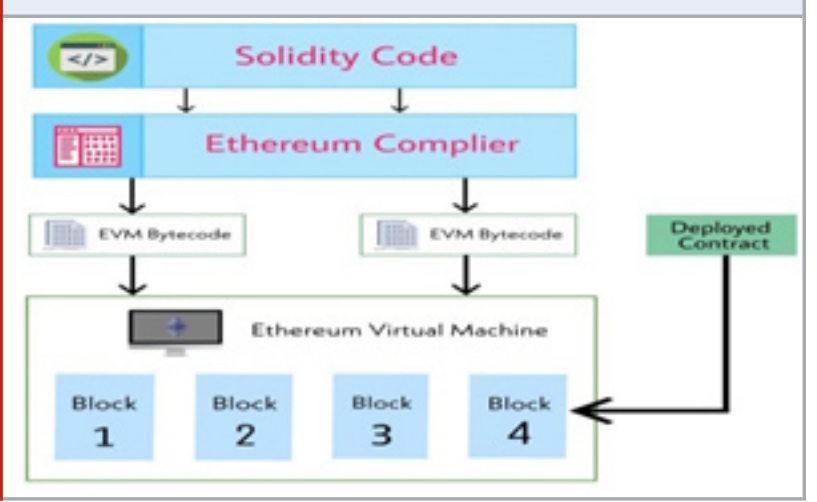

The Ethereum Blockchain is one of the distributed ledger technology, where DApps are often referred to as smart contracts. Most experts define first generation blockchains as only transacting their own native currency or token. Second generation blockchains not only manage their own native token, but also any type of asset can be defined. They can also take action on transactions with programs called Smart Contracts. Smart Contracts enforce the rules of the transaction. Smart Contracts are not legal contracts, but computer programs that are activated based on the Ethereum transactions. Ethereum Smart Contracts can be written in Solidity which is a programming language. Smart Contracts which are deployed on Ethereum blockchain are written in object-oriented Solidity programming language. Smart Contracts are compiled to the EVM (Ethereum Virtual Machine), and are deployed to the Ethereum networks.

\subsection{Blockchain Based Smart Contracts for Issuing} Driving license: The benefits of blockchain are unique to its architecture and construction. Blockchain would be the perfect solution for a transparent driving license registration system. The database of drivers and database of vehicles for different regions is maintained by the [Wikipedia-The Regional Transport Office] Regional Transport Office (RTO) also known as Regional Transport Authority (RTA). It is responsible for administrating various activities like issuing driving licences, registration of vehicles, collection of excise duty which include road tax and road fund license. The RTO also looks after selling of personalized registrations, inspection of vehicle's insurance and clearance of pollution test of vehicles.

Every region has its RTO office which governs the administration of road transport which also includes issuing of driving license. A regional RTO head is a person who administers the RTO office of that region. Several authorized agents/officers operate under his administration. However apart from these agents there are several fraud agents who sell false driving license. Thus, a fraud proof system is required to eliminate fraud agents and identify false driving licences. Blockchain technology has the ability to overcome the problems faced in the traditional system of issuing driving licences.
4. Implementation Details: Every department under RTO can be designed and implemented via blockchain smart contract system. Smart contact system in implementation will be adopted for issuing driving licences and performing verification also. The purpose of designing this smart contract is to facilitate the RTO to overcome the administrative inefficiency. This system will help in eliminating fraud agents and other middlemen and management of complex and large amount of data and procedures. Along with this, it is capable of carrying out necessary transactions which would in turn help to curb corruption and other monetary issues.

Figure 2: Smart Contract for issuing Driving licence and its verification

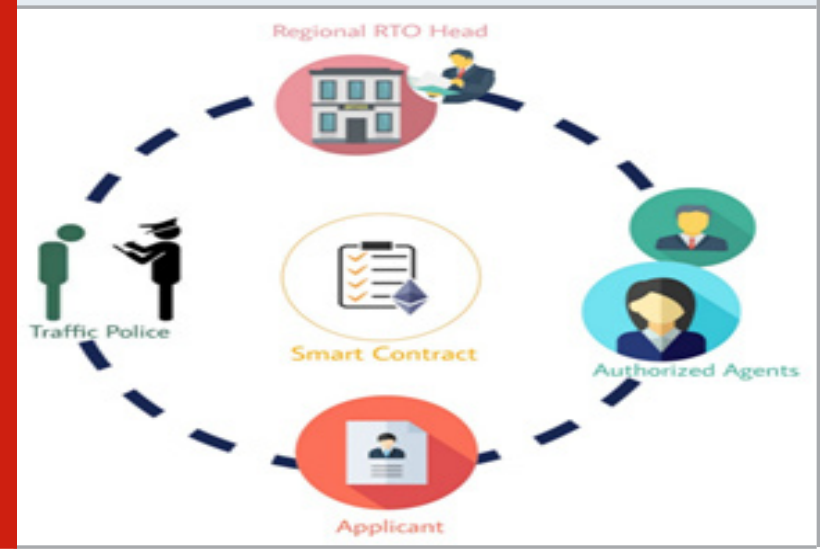

4.1 Registration of authorized agents/officers: The main goal is to streamline the process of registration of driving license and handling process by eliminating the long waiting time process, removing the fraud agents from the system and reducing the human error rate. Only the regional RTO head will have the authority to appoint agents which would be handling the process of registration and issuing new driving licenses. Thus, any agent outside the blockchain will not be able to carry out transactions in the blockchain and generate any fake license. The Ethereum Smart Contracts act as laws and thus can restrict the unauthorized agents or outsiders.

4.2 The Process for Issuing and Filling Driving Licence: In this process, metadata will be handled which includes registration of driving license via smart contract. The agents will verify the data submitted by the applicant and after verification of the data if found authorized, the authorized agents would generate and add the driving license to the blockchain.

4.3 Verifying the Driving License: Only authorized agents which were registered by the regional RTO head will have the access to view and verify the driving license via smart contract. If a driver is found unfit for driving or violating the traffic rules the agent/traffic regulator has the authority to add remark in the driver's driving license that would impose more strict restrictions on people and encourage them to follow traffic rules and thus prove helpful in reducing the road accidents. The date of issue 
and the date till which the license is validated are stored in the form of timestamp which can be converted easily using epoch time converter. With this approach, manual entry errors can be eliminated.

4.4 Other features offered by the driving license smart contact: In case of transfer/retirement of the regional RTO head the smart contract allows transfer of ownership of the smart contract to the new account and this can be done only by old regional RTO head. If an agent is found using wrong means or in case of his retirement/ transfer the regional RTO head has the authority to remove the agent.

\subsection{Potential Benefits of Blockchain in Digital Driving} License: Once driving license is registered on blockchain, then making payments for traffic violations and fine will become easier and seamless. Often, in case of higher payment fees, the license holder simply issues another new driving license, as there is no mode to check it. However, blockchain provides a mechanism for such payments. The amount of fine can be directly transferred from the license holder's account to one single address i.e. directly to the government. Once the concept of blockchain is introduced in the system of driving license, a whole new portal of innovation opens up.

\section{CONCLUSION}

In this paper, a Smart Contract based on decentralized network using blockchain technology is considered to propose an adaptive attack strategy that eliminates the frauds in driving license registration system. The Smart contract system is proposed in order to eliminate the fraud agents from the cycle of driving license registration and generation. Only genuine and eligible drivers would be allowed to drive vehicles, and the verification of the drivers via smart contract will ensure that. This smart contract opens up new opportunities for identifying people and making other process easier like renewing driving license and transacting the fines to a single account. With this system, catching criminals and fugitives will become streamlined and corruption will end.

\section{REFERENCES}

Ameer Rosic, Smart Contracts: The Blockchain Technology that Will Replace Lawyers, accessed http://www.legalserviceindia.com/legal/article-1907blockchain-technology-and-smart-contracts.html Drummond Reed, Jason Law \& Daniel Hardman, White Paper: The Technical Foundations of Sovrin, Sovrin Foundation, 29th September 2016, accessed from https:// www.evernym.com/wp-content/uploads/2017/07/TheTechnical-Foundations-of-Sovrin.pdf

Dr. Christian Lundkvist, Rouven Heck, Joel Torstensson, Zac Mitton, Michael Sena, uPort: A platform for selfsovereign identity, Draft Version (2016-10-20)

Fries, Martin; P. Paal, Boris. Smart Contracts, Mohr Siebeck. ISBN 978-3-16-156911-1.Retrieved 24 May 2020.

Michael Crosby, Pradan Pattanayak, Sanjeev Verma, Vignesh Kalyanaraman, Blockchain Technology: Beyond Bitcoin, Applied Innovation Review, Issue No.2 2016

Rajarshi Mitra, Blockchain and Digital Identity: Ultimate Guide, accessed from https://blockgeeks.com/guides/ blockchain-and-digital-identity-ultimate-guide/ Raval, Siraj (2016), "Decentralized Applications: Harnessing Bitcoin's Blockchain Technology” 0'Reilly Media, Inc. pp. 1-2. ISBN 978-1-4919-2452-5.

Roger Aitken, Blockchain To The Rescue Creating A 'New Future' For Digital Identities, Published by Forbes The Known Traveller - Unlocking the potential of digital identity for secure and seamless travel, World Economic Forum in collaboration with Accenture, January 2018, accessed from https://www.weforum.org/reports/theknown-traveller-unlocking-the-potential-of-digitalidentity-for-secure-and-seamless-travel

The Regional Transport Office, accessed from https:// en.wikipedia.org/wiki/Regional_Transport_Office Vinny Lingham, Jonathan Smith, Civic Technologies, Civic-CVC Whitepaper (2017), Identity verification via the blockchain,USA, accessed from https://icosbull. com/eng/ico/civic 